\title{
Spondyloperipheral Dysplasia
}

National Cancer Institute

\section{Source}

National Cancer Institute. Spondyloperipheral Dysplasia. NCI Thesaurus. Code C135088.

An autosomal dominant condition caused by mutation(s) in the COL2A1 gene, encoding collagen alpha-1(II) chain. It is characterized by short stature, pugilistic facies, midface hypoplasia, spondyloepiphyseal dysplasia, kyphosis, short ulna, and absent styloid process. Mutation(s) in the same gene are responsible for Kniest dysplasia. 